\section{The role of IL-33 in preventing metabolic disease}

$\operatorname{Dr}$ Amal Hasan is based at the Dasman Diabetes Institute, State of Kuwait. Her work
looks at the mechanics behind looks at the mechanics behind the relationship between obesity and metabolic disease. In particular, Dr light on the involvement a molecule called IL-33 in metabolic disease. IL-33 and its interactions with another molecule, ST2, may play role in metabolic health in humans and may represent a novel target for glucoselowering therapy.

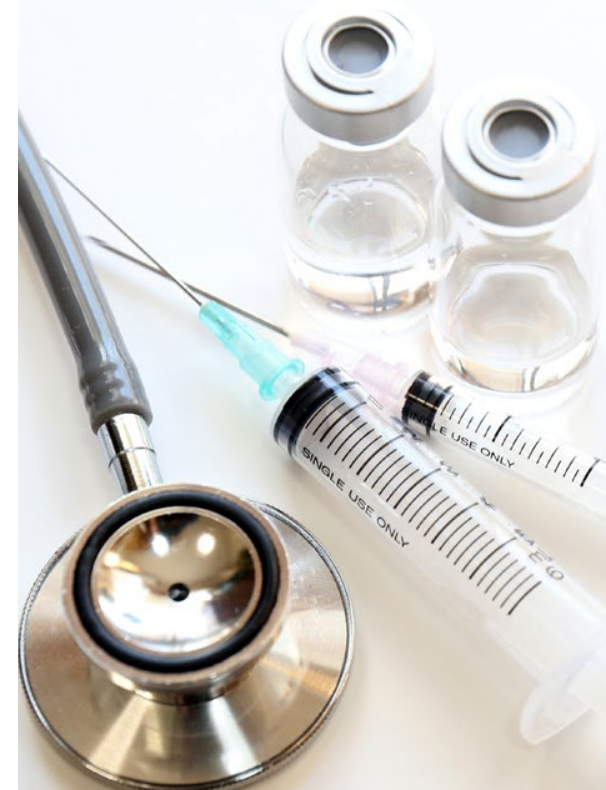

s sthe number of people with obesity rises globally, there often associated with being a higher weight. For example, there is an increased risk of developing metabolic syndrons increase risk of heart disease, stroke and type 2 diabetes. These conditions indude high blood pressure, uncontrolled high blood sugar levels, abnormal cholestero and increased waist circumference. Type 2 diabetes differs from type 1 diabetes in that it is not caused by an absolute lack of insulin production but is due to a combination of the body becoming resistant to the effects of insulin and the pancreas being unable to produce sufficient amounts of insulin to control blood sugar levels. While type 2 diabetes is normally managed using lifestyle and dietary interventions as a first line optior drugs to lower blood sugar levels are often required.

Some individuals remain metabolically healthy despite having obesity. This is contend there is no such thing as metabolically healthy obese people, and that the consequences of excess body fat will almost always be present at some point during the lifespan.

\section{INFLAMMATION AND}

METABOLIC SYNDROME

The work of Dr Amal Hasan, Dasman Diabetes Institute, State of Kuwait, looks at the link between MetS and obesity in an effort to understand what is responsible for
Excess fat accumulation and the resulting dysregulation of fat metabolism can Thduce a state of chronic inllammation. This inflammatory response wil result in excessive production of proproduction of lipid (fot) metabolites. This perfect storm of inflammation and deranged metabolism contributes to the development of diseases such as heart disease and fatty liver disease but also impacts upon insulin resistance and development of type 2 diabetes.

In particular, Dr Hasan is interested in a molecule called interleukin-33 (IL-33). IL-33 is constitutively expressed in the nucleus, or the control centre, of various types of cells, including adipocytes (fat cells) and pre-adipocytes, as well as the cells which line the inner surface of blood vessels, endothelial cells, and those which hine olher body and organ surfaces, epinela cells. In addition, IL-33 can be induced upon activation of some monocytes/macrophages, dendritic cells and mast cells. The complementary eceptor that the IL-33 molecule binds to (ST2) and this can be found in two forms; either bound to the membran of cells (ST2L), or as a soluble, secreted protein (sST2). ST2L is responsible for the biological actions of $\mathrm{LL}-33$, whereas SST2 acts as a decoy receptor, meaning that although it is able to bind IL-33, it does not activate IL-33 and thus acts as an inhibitor by preventing IL-33 binding to its activation molecule, ST2

IL-33 plays a role in type 2 immune responses, which are traditionally with parasites, but the role of IL-33

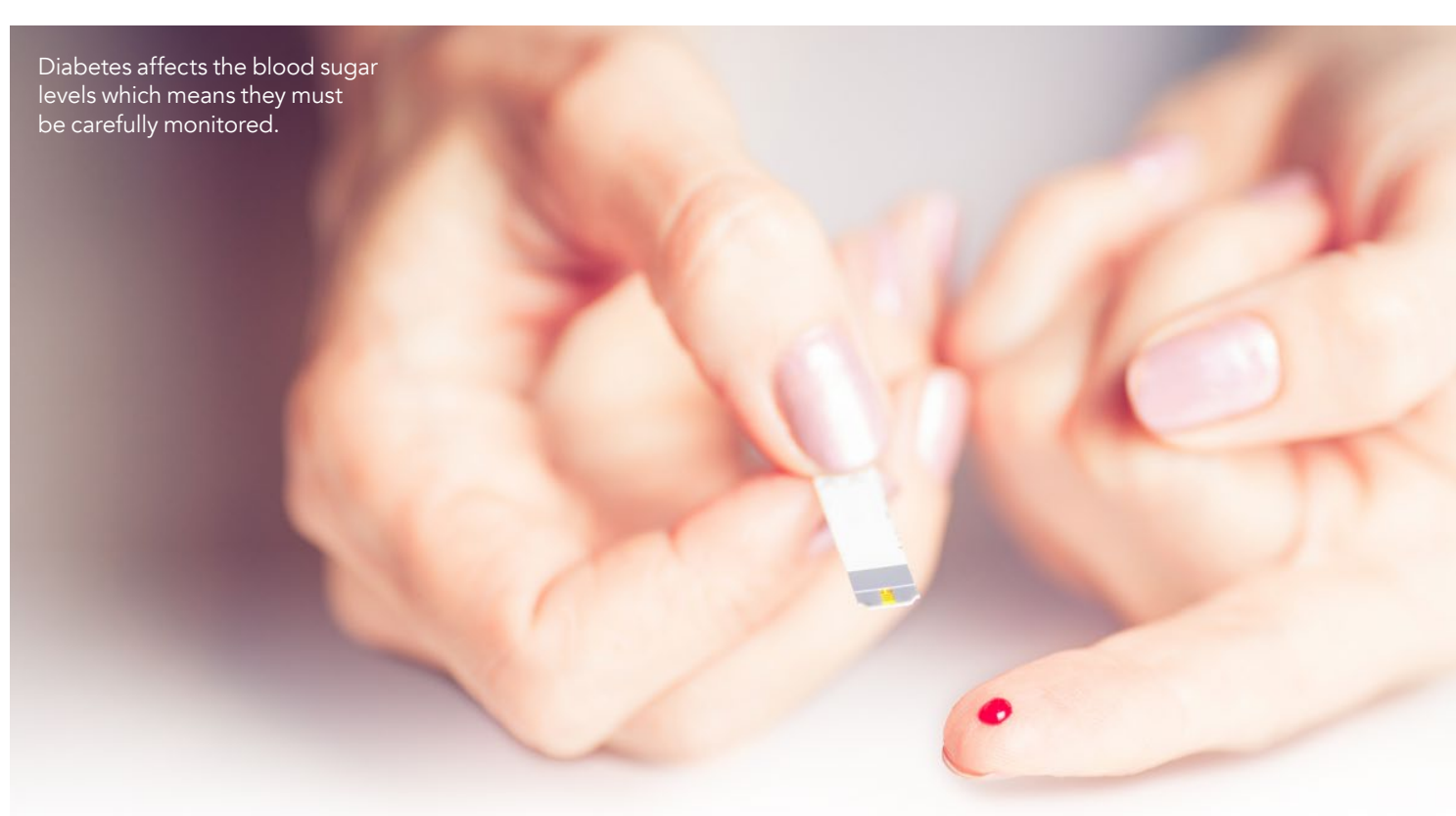

in metabolic disease is receiving increasing attention. Previous studies expression of a hunger-suppressing hormone in human fat tissue, and studies using animal models have reported that the IL-33/ST2 axis is protective against obesity and/or type 2 diabetes. IL-33 promotes the production of $\mathrm{T}$ helper type 2 (Th2) cytokines, polarises macrophages towards a protective alternatively activated phenotype, reduces lipid storage and decreases the expression of genes associated with lipid metabolism and formation of new fat cells. In addition, previous work of Dr Hasan and he peserrous woup has protective metabolic effects in humans.

three months: while it does not show daily or weekly fluctuations in blood sugar levels, it is a useful tool to get

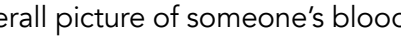
CD302 and fibrinogen-like protein 2 . There was also an association with a which is invedomain containing 16 , fat tissue, a move from the white associated with excess dietary intake of

Some individuals remain metabolically fat towards the healthy despite being obese.

at associated

inversely correlated with fasting blood glucose in individuals with type 2 diabetes who had better control of the blood sugar. Overall, these findings suggest a protective role for IL-33

In individuals who are considered metabolically 'healthy' obese, Dr Hasan IL-33 and ST2 and seition between

\section{THE ROLE OF IL-33}

\section{IN TYPE 2 DIABETES}

Dr Hasan's more recent work
investigated whether the level of IL-33 in fat tissue differs between healthy people and those diagnosed with type 2 diabetes or pre-diabetes. She found that although the level of IL-33 was not significantly different between these three groups, IL-33 was inversely associated with HoAsc in individuals with normal blood sugar levels and type 2 diabetes, but not in those with pre-diabetes. HbA1c, or glycated haemoglobin, is a measurement used to gauge the average blood sugar
In contrast to these findings, IL-33 was also inversely correlated with two was

Similar trends were observed in individuals with type 2 diabetes. additionally correlated with a pattern system, TLR3, and another chemical and called produced by activated immune

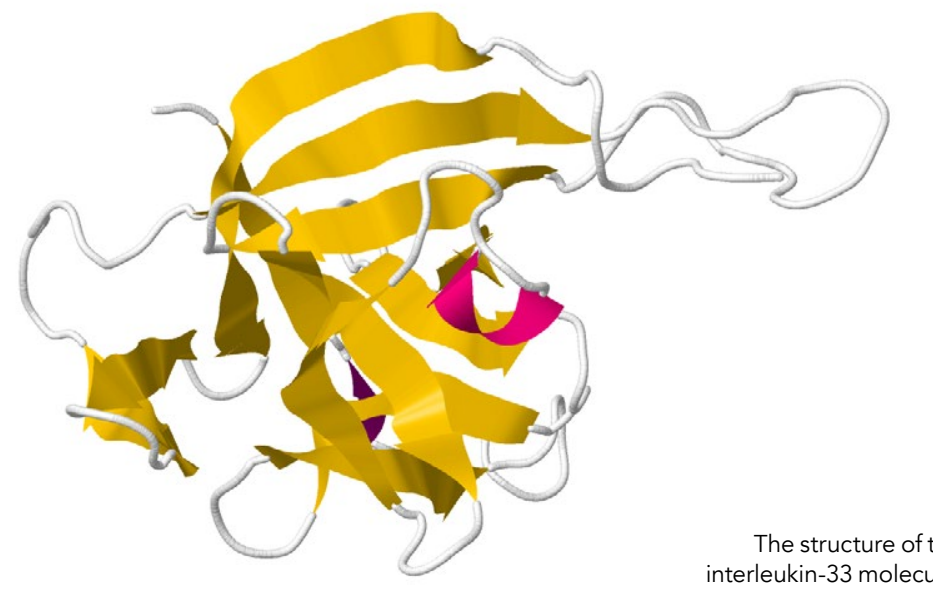
recognition receptor of the immune 
cells, called IL-12. In those with prediabetes, IL-33 did not appear to be mentioned above, but instead was strongly associated with another patt recognition receptor, which is normally activated by bacteria and viruses, TLRg.

Dr Hasan concludes that IL-33 found in fat tissue, and potentially its

interaction with ST2, may play a role in blood sugar levels. One proposed mechanism for this role is that IL-33 promotes immune responses that are protective against obesity-associated inflammation, insulin resistance and type 2 diabetes. The lack of an association between IL-33 and blood sugar levels in those with prediabetes, but notwin confrimed type 2 dabet, in the IL-33/ST2 axis in early stage metabolic disorders and its revers with glucose-lowering medications, as may be occurring in patients with type diabetes. In other words, there doesn't seem to be an immunometabolic response in those with early stage diabetes and this may be reversible.

THERAPEUTIC POTENTIAL Fat IL-33 and its interactions with ST2 may play a role in metabolic health in humans and may represent a novel target for glucose-lowering therapy. Therefore, future work will lead on from these findings and may include novel
mechanistic and clinical studies in people mechanistic and clinical studies in people
with prediabetes and type 2 diabetes.

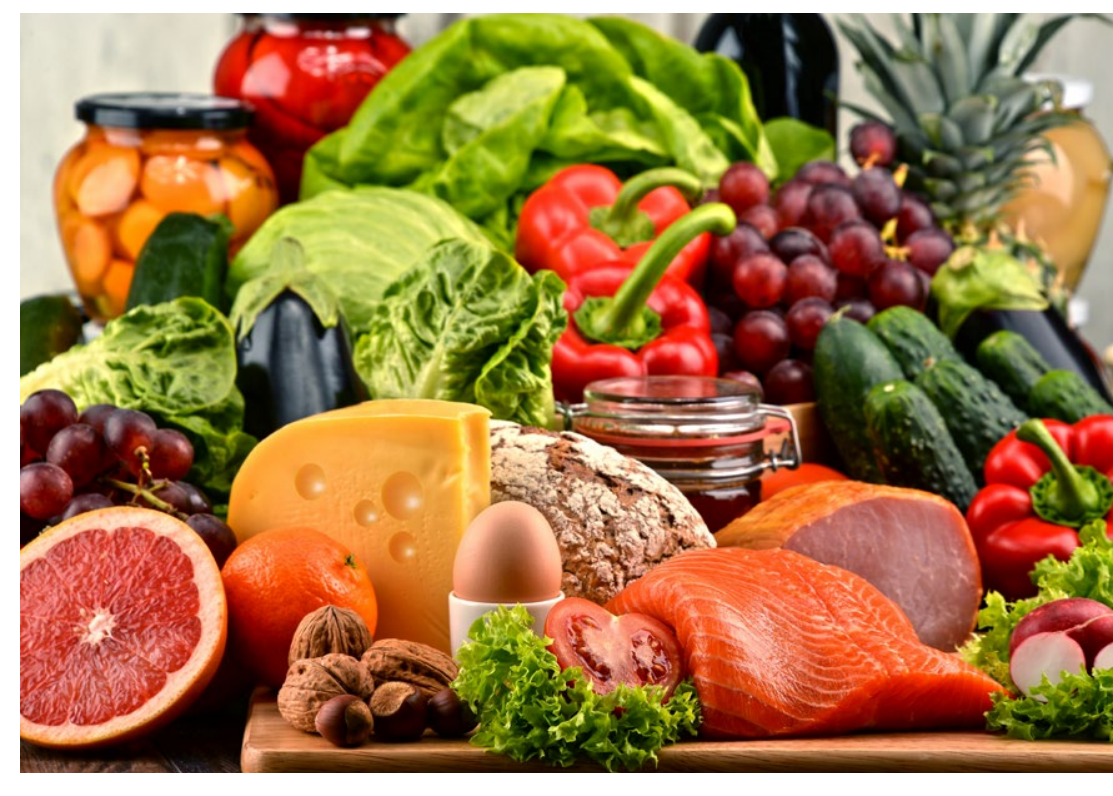

IL-33 is negatively associated with weight and confers a protective metabolic profile in non-diabetic, but not diabetic subjects.

Furthermore, exogenous administration ST2 among individuals with normal blood sugar levels may promote reversal 2 diabetes, IL-33 is inversely correlated of the metabolic derangements that lead to MetS. Dr Hasan suggests that it may be beneficial to start glucoselowering interventions in the early stages

\section{CONCLUSION}

Although there is no difference in the gene expression level of fat IL-33 or

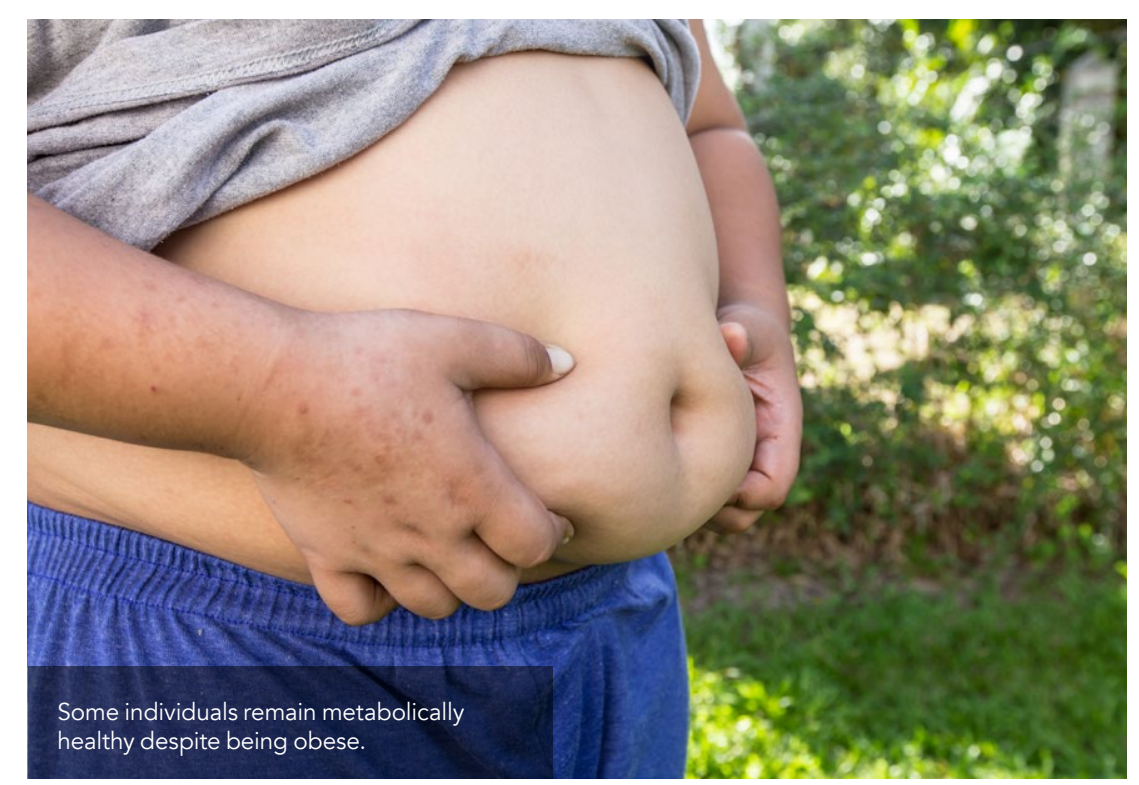

2 diabetes, L-33 is inversely correlated with but not prediabetes. In contrast, IL-33 is directly correlated with ST2 in individual with normal blood sugar levels or type 2 diabetes, but not prediabetes

When the underlying mechanism for the link between obesity and metabolic disease is considered, IL-33 is associated with mediators of immune regulation blood sugar levels and type 2 diabetes, but not prediabetes. It is also directly correlated with a mediator involved in beiging of fat in individuals with norma blood sugar levels and type 2 diabetes but not prediabetes.

Increasing understanding of the causal relationship between obesity and MetS offers the opportunity to both further knowledge and to investigate potential novel treatment options. The work of Dr Hasan will allow researchers, healthcare professionals and individuals to better understand the protective mechanisms that the hur

\section{A Behind the Research \\ Dr Amal Hasan \\ E: amal.hasan@dasmaninstitute.org T: +96522242999 Ext. $4312 \quad$ W: http:///www.dasmaninstitute.org/ W: https:///blogs.biomedcentral.com/bmcseriesblog/2014/06/13/obesity-and-new-findings-with-
interleukin-33-il-33/

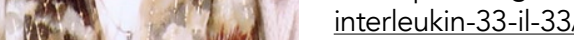

\section{Research Objectives}

Dr Hasan's work looks at the mechanics behind the relationship between obesity and metabolic disease.

\section{Detail}

Al-Soor Street

Dasman

P.O. Box 1180, DDI 15462

Kuwait

Bio

Amal Hasan is a scientist in the Department of Microbiology and Immunology at Dasman Diabetes Institute. She has a BSc in Zoology (minoring in Microbiology), an MSc in Immunology and Allergy, and a PhD in Immunology from the University of Nottingham. Before assuming a position in
Dasman Diabetes Institute, she worked in the Department of Immunology at Hamed Al-Essa Organ Transplant Centre.

Funding

The program was financially supported by the Kuwait Foundation for the Advancement of Sciences (KFAS).

Collaborators

The research group / co-authors in the publication.

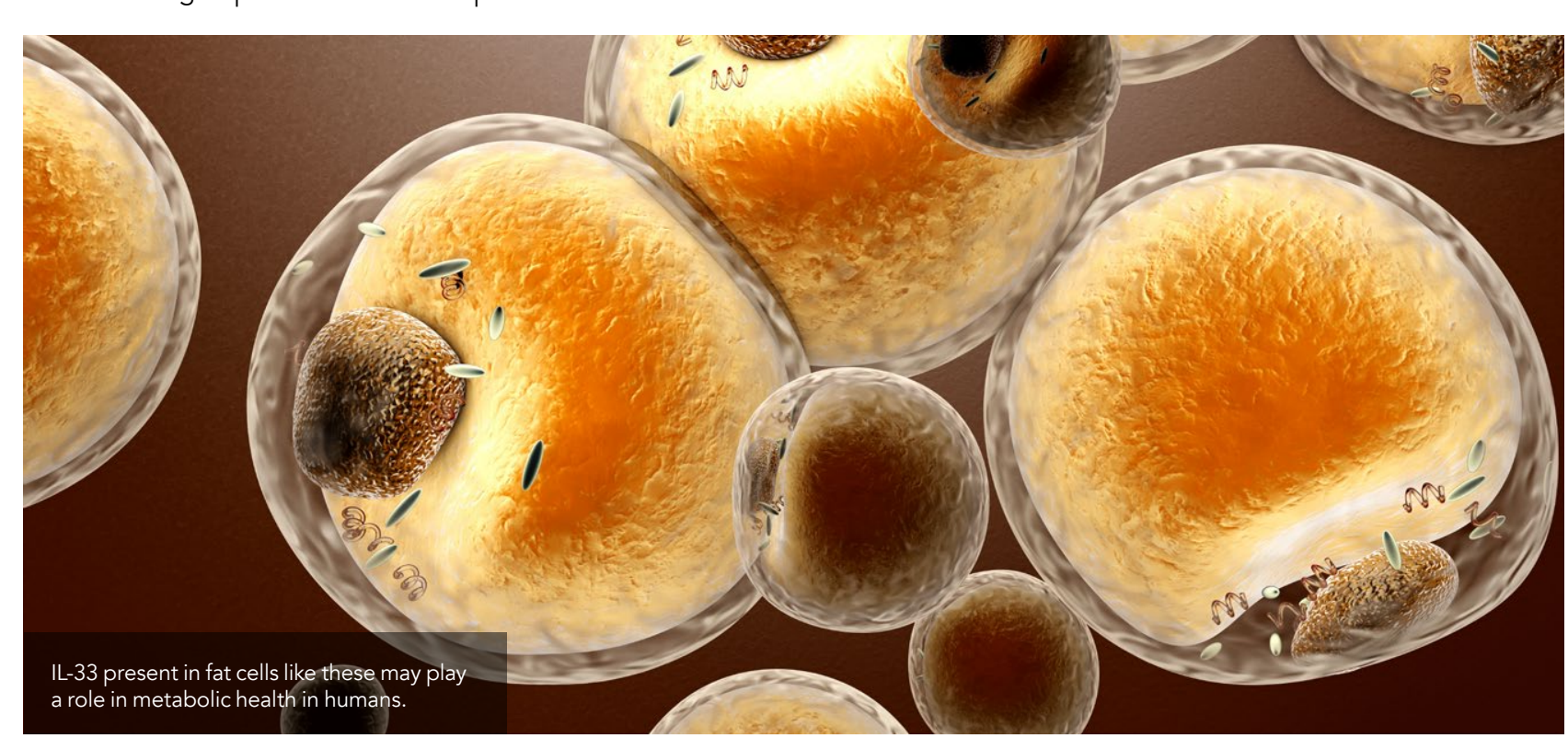

\section{References}

Hasan, A., Kochumon, S., Al-Ozairi, E., Tuomilehto, J. \& Ahmad, R. (2019). Association between Adipose Tissue with Varying Degrees of Glycemia. Disease Markers. 2019 Ahmad, R., Bennakhi, A., Al-Arouj, M., Behbehani, K., Dehbi, M \&Dermime, S. (2014). IL-33 is negatively associated with the BMl and confers a protective lipid/metabolic profile in 15(19). doi: 10.1155/2019/7901062

\section{Personal Response}

What first interested you in this area of research?

II I was intrigued by the pleiotropic nature of L-33 where it can be proinflammatory in some disease states but protective in others such as obesity and type 2 diabetes.
The IL-33/ST2 axis is metabolically protective in animal models, so I wanted to find out whether this was the case in humans. Such findings may lead to novel insigh into the pathophysiological mechanisms involved in the strategies for prevention and/or treatment.
Hasan, A., Al-Ghimlas, F., Warsame, S., Al-Hubail, A. 